\title{
Linfoma primario del sistema nervioso central en una paciente inmunocompetente. Caso clínico
}

\author{
David Coronel $F^{1 a}$, Cristián G allardo $V^{1 a}$, \\ Claudia G amargo $\mathbf{G}^{1}$.
}

\section{Primary central nervous system lymphoma in an immunocompetent patient. Report of one case}

Primary central nervous system lymphoma (PCNSL) is a rare tumor. It occurs mainly in people aged 50 year-old or older and is more common among men. Immunodeficiency is the only established risk factor for PCNSL. We report a 35 year-old, immunocompetent woman who presented with a two weeks history of persistent headache. Computed tomography (CT) and magnetic resonance imaging (MRI) showed an expansive lesion in the right thalamus area. Immunohistochemical studies were consistent with the diagnosis of a difuse large B-cell non-Hodgkin's lymphoma. The patient was treated with chemotherapy and whole brain radiotherapy, achieving complete remission of the tumor. This case is ilustrative of PCNSL and contributes to update its diagnosis, management and prognosis (Rev Méd Chile 2008; 136: 491-5).

(Key words: Antineoplastic protocols; Brain neoplasms; Lymphoma, non-Hodgkin)

Recibido el 29 de diciembre, 2006. Aceptado el 27 de julio, 2007.

${ }^{1}$ Departamento de Oncología, Hospital Barros Luco Trudeau. Santiago de Chile.

${ }^{a}$ Interno, Facultad de Medicina, Clínica Alemana - Universidad del Desarrollo.

$\mathrm{E}$ 1 linfoma primario del sistema nervioso central (LPSNC) es un subtipo poco frecuente de linfoma no Hodgkin (LNH) que se origina en el cerebro, ojos, leptomeninges o médula espinal ${ }^{1}$. Da cuenta de $3 \%$ de los tumores primarios del $\mathrm{SNC}^{2}$ y $1 \%-2 \%$ de todos los linfomas extranodales $^{3}$. El único factor de riesgo establecido para desarrollar LPSNC es la inmunodeficiencia, congénita o adquirida. Personas con síndrome de inmunodeficiencia adquirida (SIDA) tienen incre-

Correspondencia a: David Coronel F. Jesús 705, casa 6. La Reina, Santiago, Chile. Fonos: 02-2660860 - 08-4294350. E mail: dcoronelf@gmail.com mentado el riesgo de padecer LPSNC en 3.600 veces comparado con la población general ${ }^{4}$.

Comunicamos un caso de LPSNC difuso de células grandes B en un huésped inmunocompetente.

\section{Caso Clínico}

Mujer de 35 años de edad con antecedente de una tiroidectomía subtotal bilateral hacía 6 años, por bocio difuso hipertiroideo con mala respuesta a tratamiento médico, el día 13/12/2005 presentó un cuadro caracterizado por inicio súbito de cefalea holocránea y cervicalgia, asociado a obnubilación mental de segundos de duración. Desde esa fecha 
evolucionó con cefalea persistente, lo cual la llevó a consultar al Servicio de Urgencia el día 02/01/2006.

$\mathrm{Al}$ ingreso al Servicio de Urgencia se encontraba afebril, hemodinámicamente estable, con un examen neurológico normal, fondo de ojo normal y $\sin$ historia de baja de peso. Se realizó una tomografía computada (TC) cerebral que reveló un proceso expansivo a nivel talámico derecho de $3,5 \mathrm{~cm}$ de diámetro. Se decidió hospitalizar. Se efectuó una resonancia magnética (RM) cerebral que confirmó la lesión expansiva talámica, con significativo edema perilesional y con mínimo efecto de masa sobre las estructuras de la línea media. Ambas imágenes (TC y RM) se reforzaron significativamente luego del medio de contraste, captándolo en forma heterogénea (Figura 1).

La TC de tórax, abdomen y pelvis no presentaban alteraciones. La biopsia de médula ósea estaba dentro de límites normales. La punción lumbar (PL) y posterior citometría de flujo evidenció líquido cefalorraquídeo (LCR) sin células linfomatosas y con proteína en rangos normales. Recuento sanguíneo y exámenes de laboratorio sin anormalidades, excepto por el ácido láctico moderadamente elevado $(23,6 \mathrm{mg} / \mathrm{dl}$, rango normal 4,5-18 mg/dl). Virus de inmunodeficiencia humano (VIH) negativo. El 30/01/2006 se realizó una biopsia estereotáxica de la lesión. Los resultados, disponibles el día 03/03/2006, fueron compatibles morfológica e inmunohistoquímicamente con un LNH difuso de células grandes B. Los anticuerpos monoclonales en las células tumorales fueron negativos para fosfatasa alcalina placentaria (PLAP) y CD 3 (Pan-T) e intensamente positivos para CD 20 (Pan-B) (Figura 2).

La inmunohistoquímica asociada a un examen de extensión negativo permitió diagnosticar un LPSNC difuso de células grandes B.

A la espera de los resultados de la inmunohistoquímica y sólo con tratamiento sintomático para su cefalea, con paracetamol $500 \mathrm{mg} / 8 \mathrm{~h}$, la paciente no acude a sus controles preestablecidos, consultando el día 29/03/2006 por síndrome piramidal a izquierda (cuadro de 2 semanas de evolución con alteración del equilibrio, paresia braquio crural izquierda armónica, reflejos osteotendíneos algo aumentados en dicha distribución y reflejo plantar extensor a izquierda), disminu-

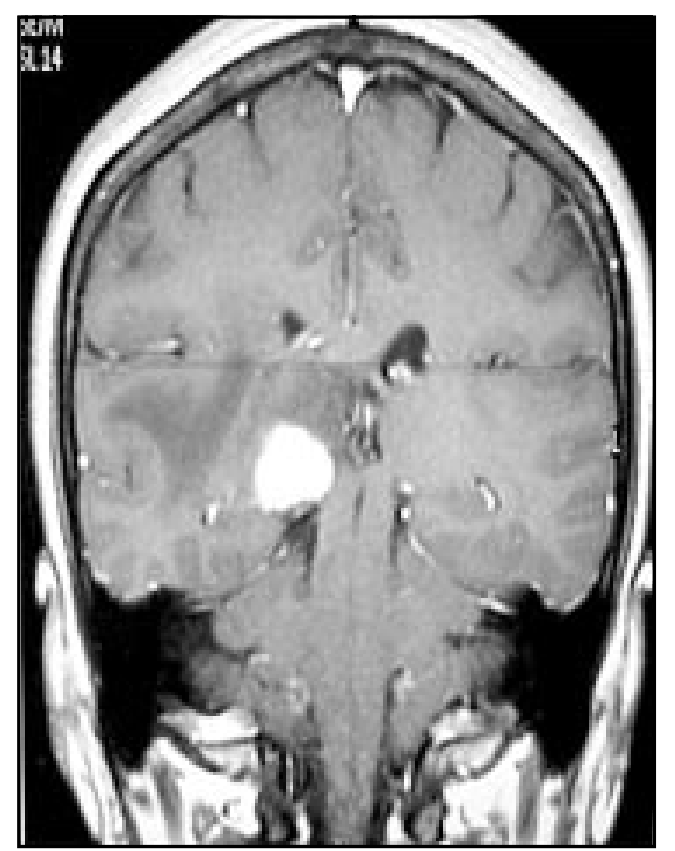

Figura 1. Corte coronal en secuencia SE potenciada en T1 tras la administración de gadolinio: se identifica una lesión hiperintensa a nivel talámico derecho, con mínimo efecto de masa sobre las estructuras de la línea media, que a su vez presenta un realce significativo tras la administración de contraste. 


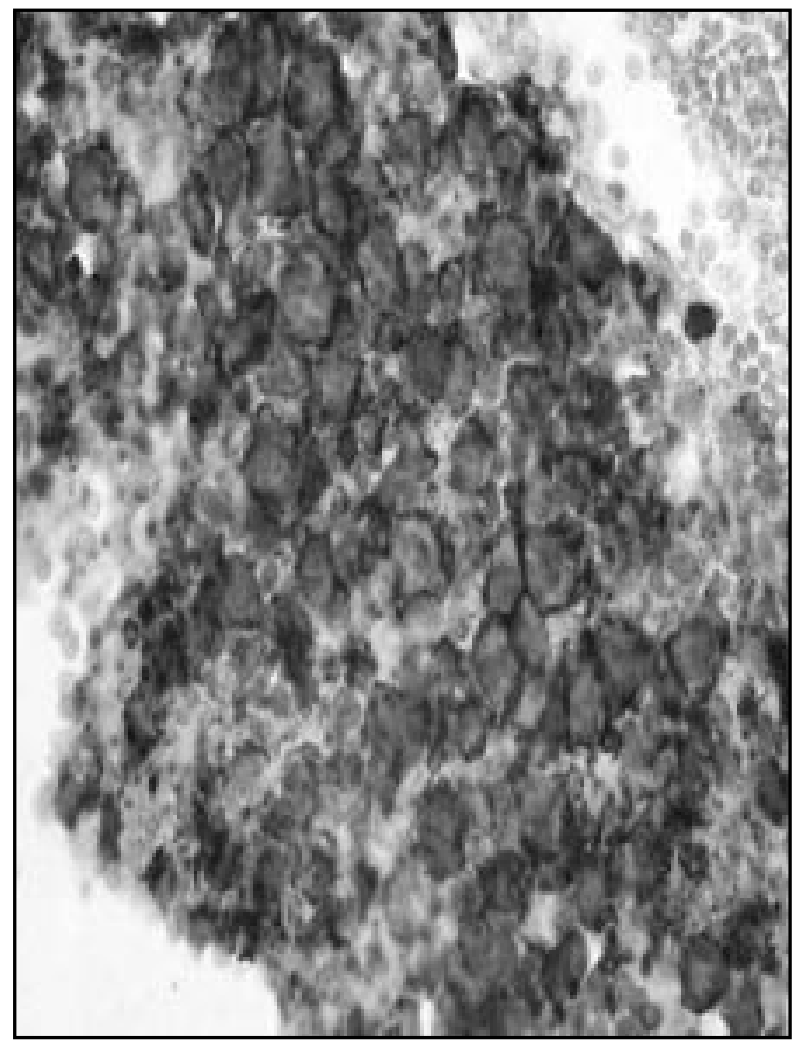

Figura 2. Microfotografía del tumor cerebral con tinción de CD 20 (400x). Los linfocitos tumorales expresan el antígeno CD 20 en su membrana, lo cual es marcador de linfocitos inmunofenotipo B.

ción de la agudeza visual del ojo izquierdo y cefalea holocránea persistente, por lo cual fue hospitalizada. Se inició quimioterapia según protocolo del hospital con metotrexato endovenoso $1,8 \mathrm{~g} /$ día los días 30/03/06 y 06/04/06, ácido folínico vía oral $15 \mathrm{mg} / 6 \mathrm{~h}$ los días 31/03/06 y 01/ 04/06, y dexametasona endovenosa $16 \mathrm{mg} /$ día desde el día 30/03/06 hasta el 13/04/06. Evolucionó favorablemente, resolviéndose el síndrome piramidal, la disminución de la visión y la cefalea, por lo cual fue dada de alta luego de 15 días hospitalizada. En una TC cerebral de control 2 semanas posteriores al alta se observó franca regresión de la masa, pero aún con persistencia de hipodensidad. Se aplicó radioterapia externa holocraneal 3850 cGy con fraccionamiento estándar de 180 cGy/día, 5 sesiones/semana, desde el 05/06/06 hasta el 20/07/06. La valoración final de respuesta mediante TC, el 24/08/06, fue de remisión completa radiológica. Inicialmente se le hizo seguimiento mensual, para luego ir distanciando los controles en relación con su evolución favorable. En su último control, el 21/03/07, la paciente se encontraba en buen estado general, hemodinámicamente estable, sin síntomas ni signos de focalización neurológica y con hemograma y perfil bioquímico en rangos normales.

\section{DisCUSIÓN}

El LPSNC es un LNH agresivo, causado por un linfoma difuso de células grandes B en aproximadamente $90 \%$ de los casos ${ }^{5}$. Resultan indistinguibles microscópica e inmunológicamente de los LNH sistémicos ${ }^{6}$. Se desconoce por qué se desarrolla primariamente en un órgano que carece de sistema linfático, aunque se piensa que es debido a un neurotropismo específico ${ }^{7}$. Su incidencia es $2 \%$ de todos los linfomas malignos y $3 \%$ de los tumores primarios del $\mathrm{SNC}^{1}$. Afectan más frecuentemente a los varones con una razón de 1,2:1 a 1,7:1 y su máxima incidencia se sitúa en la sexta década de la vida $^{8}$. Las condiciones asociadas a inmunodepresión aumentan el riesgo de padecer un linfoma primario del $\mathrm{SNC}^{3}$.

La forma clínica de aparición más frecuente es la focalidad neurológica (70\%), seguida por síntomas neuropsiquiátricos (43\%), hipertensión intracraneana (33\%), crisis epilépticas (14\%) y síntomas oculares como visión borrosa, disminución de la agudeza visual y ojo rojo doloroso $(4 \%)^{9}$. Las lesiones son solitarias en $65 \%$ de los pacientes y multifocales en $35 \%$. Se localizan en los hemisferios (38\%), tálamo/ganglios basales (16\%), cuerpo calloso (14\%), región ventricular $(12 \%)$ y cerebelo $(9 \%)^{10}$. La mayoría de las lesiones se ven isodensas o hiperdensas en la TC (92\%), e isointensas o hipointensas en la RM en T2. Casi todas las lesiones captan contraste homogéneamente, excepto tras la administración de corticoides, los cuales pueden ser oncolíticos, haciendo desaparecer el tumor (tumores fantasma). Pueden presentar edema perilesional y efecto de masa ${ }^{11}$. Los LPSNC se presentan mayoritariamente como lesiones intraparenquima- 
tosas, en contraste con los linfomas secundarios que tienden a invadir las leptomeninges. La distribución periventricular con compromiso del epéndimo también favorece el diagnóstico de LPSNC $^{12}$. En la histología, las células linfoides se disponen típicamente perivasculares (en los espacios de Virchow-Robin) y aunque esto no es constante, es altamente específico ${ }^{13}$. Aunque se trata de masas bien definidas, no es raro encontrar invasión tumoral más allá de los márgenes macroscópicos $^{14}$.

Al momento del diagnóstico, tal como se efectuó en nuestro paciente, lo primordial es diferenciar si es un LPSNC o es un tumor secundario, para ello todos los pacientes debieran tener un acabado examen médico, neurológico y oftalmológico que incluya fondo de ojo; RM cerebral con medio de contraste, TC de tórax, abdomen y pelvis; punción lumbar para citología y citometría de flujo; biopsia de médula ósea; ecografía testicular; hemograma, electrolitos plasmáticos, lactato deshidrogenasa sérica (LDH) y serología para VIH; clearence de creatinina en orina de $24 \mathrm{~h}$ a quienes serán sometidos a quimioterapia con metotrexato; y por último, biopsia estereotáxica de la lesión para confirmación histopatológica e inmunofenotipificación ${ }^{15}$.

En relación con el pronóstico, se han encontrado 5 variables que afectan negativamente la sobrevida: edad mayor a 60 años, performance status mayor a 1, niveles séricos de LDH elevados, hiperproteinorraquia y la afectación de estructuras

\section{ReFERENCIAS}

1. Singh AD, Lewis H, Schachat AP. Primary lymphoma of the central nervous system. Ophthalmol Clin North Am 2005; 18: 199-207.

2. Batara JF, Grossman SA. Primary central nervous system lymphomas. Curr Opin Neurol 2003; 16: 671-5.

3. Fine HA, Mayer RJ. Primary central nervous system lymphoma. Ann Intern Med 1993; 119: 1093-104.

4. Cote TR, Manns A, Hardy CR, Yelun FJ, Hartge P. Epidemiology of brain lymphoma among people with or without acquired immunodeficiency syndrome. AIDS/Cancer Study Group. J Natl Cancer Inst 1996; 88: 675-9. cerebrales profundas (regiones periventriculares, ganglios basales, troncoencéfalo y cerebelo). Pacientes con 0-1, 2-3, ó 4-5 de esos factores de riesgo, tienen una sobrevida de $80 \%$, 48\% o 15\%, respectivamente ${ }^{16}$. Por contraparte, se ha visto una asociación estadísticamente significativa entre la expresión tumoral del antígeno BCL-6 y una mayor sobrevida (mediana de 101 meses de sobrevida versus 14,7 meses en quienes no lo expresan) ${ }^{17}$.

En relación al tratamiento, los esquemas más efectivos son los basados en altas dosis de metotrexato $\left(3,5 \mathrm{~g} / \mathrm{m}^{2}\right)$ seguidas de radioterapia holocraneal (estándar 45 Gy), con lo cual se logra una tasa global de respuesta de 94\%, una RC de $87 \%$ y una sobrevida media de 60 meses $^{18}$. El gran inconveniente de la terapia son las altas tasas de neurotoxicidad, especialmente en los mayores de 60 años (hasta 100\% versus sólo 30\% de los menores de 60 años al usar radioterapia $)^{19}$. La sobrevida media luego de diagnosticada la neurotoxicidad es de 12 meses y la mayoría fallece por complicaciones asociadas a ésta y no por linfoma activo $^{7}$. Por lo anterior, se están intentando regímenes terapéuticos basados únicamente en quimioterapia, con lo cual hay reportes de estudios multicéntricos en fase II, que utilizando como único agente altas dosis de metotrexato $\left(8 \mathrm{~g} / \mathrm{m}^{2}\right)$, se obtienen tasas de remisión completa de 52\%, sobrevida libre de enfermedad de 12,8 meses y sobrevida global de 55,4 meses, con mínima toxicidad secundaria ${ }^{20}$.

5. Miller DC, Hochberg FH, Harris NL, Gruber M, Louss DN, CoHen H. Pathology with clinical correlations of primary central nervous system non-Hodgkin's lymphoma. Cancer 1994; 74 : 1383-97.

6. Zazpe I, De llano P, Gorosquieta A, Cabada T, Tunon T, VázQuez A et al. Primary CNS Lymphoma: bibliographical review and experience at the Hospital of Navarre in the last 5 years (20002004). An Sist Sanit Navar 2005; 28: 367-77.

7. Batchelor T, Loeffler JS. Primary CNS lymphoma. J Clin Oncol 2006; 24: 1281-8.

8. Schabet M. Epidemiology of primary CNS lymphoma. J Neurooncol 1999; 43: 199-201.

9. Bataille B, Delwail V, Menet E, Vandermarce P, Ingrand P, Wager M et al. Primary intracerebral 
malignant lymphoma: report of 248 cases. J Neurosurg 2000; 92: 261-6.

10. Kuker W, Nagele T, Korfel A, Heckl S, Thiel E, BAMBERG M ET al. Primary central nervous system lymphomas (PCNSL): MRI features at presentation in 100 patients. J Neurooncol 2005; 72: 169-77.

11. Coulon A, Lafitte F, Hoang-Xuan K, MartinDuverneuil N, Mokhtari K, Blustajn J et al. Radiographic findings in 37 cases of primary CNS lymphoma in immunocompetent patients. Eur Radiol 2002; 12: 329-40.

12. Erdag N, Bhorade RM, Alberico RA, Yousuf N, Patel MR. Primary lymphoma of the central nervous system: typical and atypical CT and MR imaging appearances. AJR Am J Roentgenol 2001; 176: 1319-26.

13. Atlas SW, Lavi E, Fisher PG. Intraaxial brain tumors, in Atlas SW (ed): Magnetic Resonance Imaging of the Brain and Spine ( $3^{\text {rd }}$ ed). Philadelphia, PA: Lippincott Williams \& Wilkins, 2002, pp. 565-693.

14. Guinto G, Felix I, Arechiga N, Arteaga V, Kovacs K. PCNSL in immunocompetent patients. Histol Histopathol 2004; 19: 963-972.

15. Abrey le, Batchelor TT, Ferreri AJ, GospodaROwicz M, Pulczynski EJ, ZucCa E et al. Report of an international workshop to standardize baseline evaluation and response criteria for primary CNS lymphoma. J Clin Oncol 2005; 23: 5034-43.

16. Ferreri aj, Blay Jy, Reni M, Pasini F, Spina M, Ambrosetti A et al. Prognostic Scoring System for Primary CNS Lymphomas: The International Extranodal Lymphoma Study Group Experience. J Clin Oncol 2003; 21: 266-72.

17. Braaten KM, Betensky RA, de Leval L, Okada Y, Hochberg FH, Louss DN et al. BCL-6 expression predicts improved survival in patients with primary central nervous system lymphoma. Clin Cancer Res 2003; 9: 1063-9.

18. Abrey Le, Yahalom J, De Angelis LM. Treatment for primary CNS lymphoma: the next step. J Clin Oncol 2000; 18: 3144-50.

19. Abrey Le, De Angelis LM, Yahalom J. Long-term survival in primary CNS lymphoma. J Clin Oncol 1998; 16: 859-63.

20. Batchelor t, Carson K, O'Neill A, Grossman SA, Alavi J, New P et al. Treatment of primary CNS lymphoma with methotrexate and deferred radiotherapy: a report of NABTT 96-07. J Clin Oncol 2003; 21: 1044-9. 